\title{
The Diagnostic Value of P53 and Desmin in the Differentiation between Reactive Mesothelial Cells and Metastatic Adenocarcinoma in Pleural Effusion
}

\author{
Nooshin afshar Moghadam ${ }^{1}$, Behnaz Sabaghi*1, Mohammad Emami Ardestani², Mohammad Hashemi \\ Bahramani $^{1}$, Masumeh Raoufi ${ }^{3}$ and Ashkan Zarnaghian ${ }^{1}$ \\ ${ }^{1}$ Department of pathology, Iran \\ ${ }^{2}$ Department of internal medicine, Iran \\ ${ }^{3}$ Department of radiology, Iran \\ *Corresponding author: Behnaz Sabaghi, Pathology resident, Department of pathology, School of medicine, Isfahan university of \\ medical sciences, Isfahan, Iran
}

\begin{abstract}
ARTICLE INFO
Received: 幽 May 10, 2019

Published: 閩 May 17, 2019

Citation: Nooshin afshar M, Behnaz S, Mohammad Emami A, Mohammad Hashemi B, Masumeh R, Ashkan Z. The Diagnostic Value of P53 and Desmin in the Differentiation between Reactive Mesothelial Cells and Metastatic Adenocarcinoma in Pleural Effusion. Biomed J Sci \& Tech Res 18(1)-2019. BJSTR. MS.ID.003107.
\end{abstract}

Keywords: P53 Antibody; Desmin Antibody; Metastatic Adenocarcinoma; Reactive Mesothelial Cell; Pleural Effusion

\section{ABSTRACT}

Background: It is very important to differentiate between metastatic adenocarcinoma and reactive mesthelial cells in pleural effusions especially overlapping cases which differentiation is critical. We worked on Differentiating power of p53 and desmin in distinguishing between reactive mesothelial cells and metastatic adenocarcinoma in pleural effusion.

Methods: This cross-sectional descriptive-analytic study will be carried out in the pathology department of Al-Zahra Hospital. The population studied is women and men over the age of 40 who are under the tap due to their presence in the pleura area. These fluids are routinely loaded with no preservatives, and from them cell block samples are provided.

Results: The present study was performed on 64 patients with pleural effusion. They were assigned to two groups: Group I: 32 effusions containing reactive mesothelial cells; Group II: 32 effusions containing carcinoma cells. Immunostaining with p53 and desmin were performed using an Envision technique. The calculated mean values (in percentile) for stained cells for p 53 in adenocarcinoma and reactive mesothelial cells were $100 \%$ and $3.1 \%$, respectively $(\mathrm{P}=0.001)$. weakly positive and strongly positive staining for p53 in adenocarcinoma cells were respectively 10 case $(31.3 \%)$ and 22 cases $(68.7 \%)$ and in reactive mesothelial cells, these were 1 case $(3.1 \%)$ and $0(0 \%)$ respectively. The calculated mean values (in percentile) for stained cells for desmin in adenocarcinoma and reactive mesothelial cells were $21.9 \%$ and $81.3 \%$, respectively $(\mathrm{P}=$ 0.001 ). weakly positive and strongly positive staining for desmin in adenocarcinoma cells were respectively 5 cases $(15.6 \%)$ and 2 cases $(6.3 \%)$ and in reactive mesothelial cells, these were 3 cases $(9.4 \%)$ and 23 cases $(71.9 \%)$ respectively.

Conclusion: The staining pattern and intensity for p53 and desmin are useful panel for differentiation of adenocarcinoma and mesothelial cells.

\section{Introduction}

Mesothelial cells are specific cells in serous cavities; when serous membranes stayed on process of inflammation or long time in effusion, these cells change in nucleus and cytoplasm feature. In some cases, morphological differentiation between reactive mesothelial cells and adenocarcinoma in serous effusions is extremely difficult [1]. One the other hand some serotypes have a malignant origin and determining the etiology of making effective therapeutic interventions and finding malignancy is more important. It is very difficult to differentiate between metastatic and reactive adenocarcinoma pleural effusions in serous fluids. A 
cytological examination of serum fluids is performed to diagnose the cancers involved in the peritoneum or pericardial spaces. About $75 \%$ of the malignant tumors are in the pleural metastasis of carcinomas [2,3]. A malignant pleural fluid is the first to see the presence of cancer in $46 \%$ of patients. The most common malignancies, such as pulmonary, breast, and gastrointestinal tumors, are the most common metastasis to serous fluids, which are more common in adenocarcinoma.

Metastatic carcinoma cells can have a wide variety in shape, which leads to difficult differentiation between mesothelial cell and adenocarcinoma cells in some cases. Often, when reactive cells are mixed with tumor cells in a cytological sample, the use of immunohistochemistry markers can help to differentiate them [4]. In order to avoid false diagnosis, the interpretation of immunohistochemical responses can help differentiate them and interpret the immunohistochemical response in each cell based on conventional cytology analysis. Immunocytochemistry can be done in samples such as flow cytometry [5]. Although, the differentiation is based on the usual morphological findings and history of the patient in terms of the presence or absence underlying malignancy [6]. In $15 \%$ of cases, the differentiation of mesothelial reactive cells from adenocarcinoma is not possible based on simple morphology. On the other hand, the person may not mention a history of primary malignancy and the positive posture is the first manifestation of adenocarcinoma. TP53 is one of the most frequently mutated genes in human cancers.

The most common mutations are single base substitutions that alter protein function. Some of the mutations being oncogenic confer gain-of-function properties [7]. Somatic mutations at specific residues have been associated with specific clinical phenotypes in different type of cancer. In addition to being frequently mutated in cancers, the gene is also highly polymorphic. The fact that some germline variants in the gene may modulate the individual susceptibility to develop cancer has evinced particular interest for the potential use as predictive markers [8]. In recent studies, immunoreactive antibodies against mesothelial cells have been introduced, which show no reaction with carcinoma cells. Recommended mesothelial markers include Calretinin, thrombomodulin, cytokeratin5/6, and HBME1 [9-16]. Therefore, the aim of this study is using immunohistochemistry to distinguish reactive mesothelial cells from metastatic adenocarcinoma in pleural effusions.

\section{Methods and Materials}

This cross-sectional descriptive-analytic study will be carried out in the cytology department of Al-Zahra Hospital. The population studied is women and men over the age of 40 who are under the tap due to their presence in the pleura area (Approval code:395975). These fluids are routinely loaded with no preservatives, and from them cell block samples are provided as follows: The liquid was centrifuged for 5 minutes at 2000 rounds, the supernatant was discharged, and 3 drops of plasma were added to the 3-drop of thromboplastin, so the specimen was coagulated. Then, put in a filter paper and enter the formalin and tissue processing machine, then slide and stack H \& E (hematoxylin-eosin). Slides are evaluated by a researcher and counselor with a few microscopes for choosing to colonize immunocytochemistry. Examples of immunocytochemistry staining include two categories: A bunch of malignant tumors that have definite malignant criteria for cytomorphology (i.e., cell cluster, prostate cancer and clear nuclear and polymorphism), as well as confirmed pathology. The second group consists of negative mimics that have a proliferated mesothelium and lack malignant creatinine, either cytologically or clinically or in terms of imaging findings.

\section{Results}

The present study was performed on 64 patients with pleural effusion. The mean age of the subjects in adenocarcinoma cells and mesothelial cells were $61.40 \pm 14.13$ years and $70.96 \pm 13.59$ years. Most people in the adenocarcinoma cells group were female, and in the Reactive mesothelial cells group were male. Among the subjects in the adenocarcinoma cells group, $21.8 \%$ of the subjects were desmin positive and 81.1 in the Reactive mesothelial cells group. And about p53,100\% of the subjects were positive staining and 3.1in the Reactive mesothelial cells group (Table 1). In the adenocarcinoma cells group, patients have underlying conditions including: Lung cancer, breasr cancer, Ovarian cancer, Gastric cancer, Bladder cancer and unknown origin (Pie chart 1). In the Reactive mesothelial cells group, patients had other underlying conditions such as PTE, DVT and COPD (Pie chart 2). Patterns of p53 and Desmin staining are shown in Figures $1 \& 2$.

Table 1: Desmin and P53 status in each group of study.

\begin{tabular}{|c|c|c|c|c|c|c|}
\hline \multirow{2}{*}{ Variable } & \multicolumn{3}{|c|}{ Adenocarcinoma cells } & \multicolumn{3}{c|}{ Reactive mesothelial cells } \\
\cline { 2 - 7 } & Negative & Weakly positive & Strongly Positive & Negative & Weakly positive & Strongly Positive \\
\hline Desmin & $25(78.1)$ & $5(15.6)$ & $2(6.3)$ & $6(18.8)$ & $3(9.4)$ & $23(71.9)$ \\
\hline P53 & 0 & $10(31.3)$ & $22(68.8)$ & $31(96.9)$ & $1(3.1)$ & 0 \\
\hline
\end{tabular}




\section{Distribution of adenocarcinoma group}

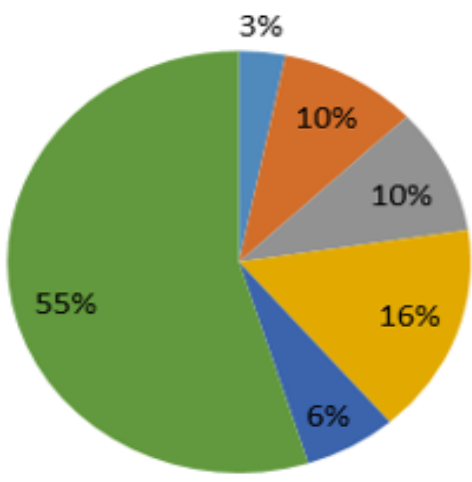

- Bladder cancer

n Breast cancer

Gastric cancer

En Lung cancer

- Ovarian cancer

- Unknown origin

Pie chart 1: Baseline disease distribution in adenocarcinoma group.

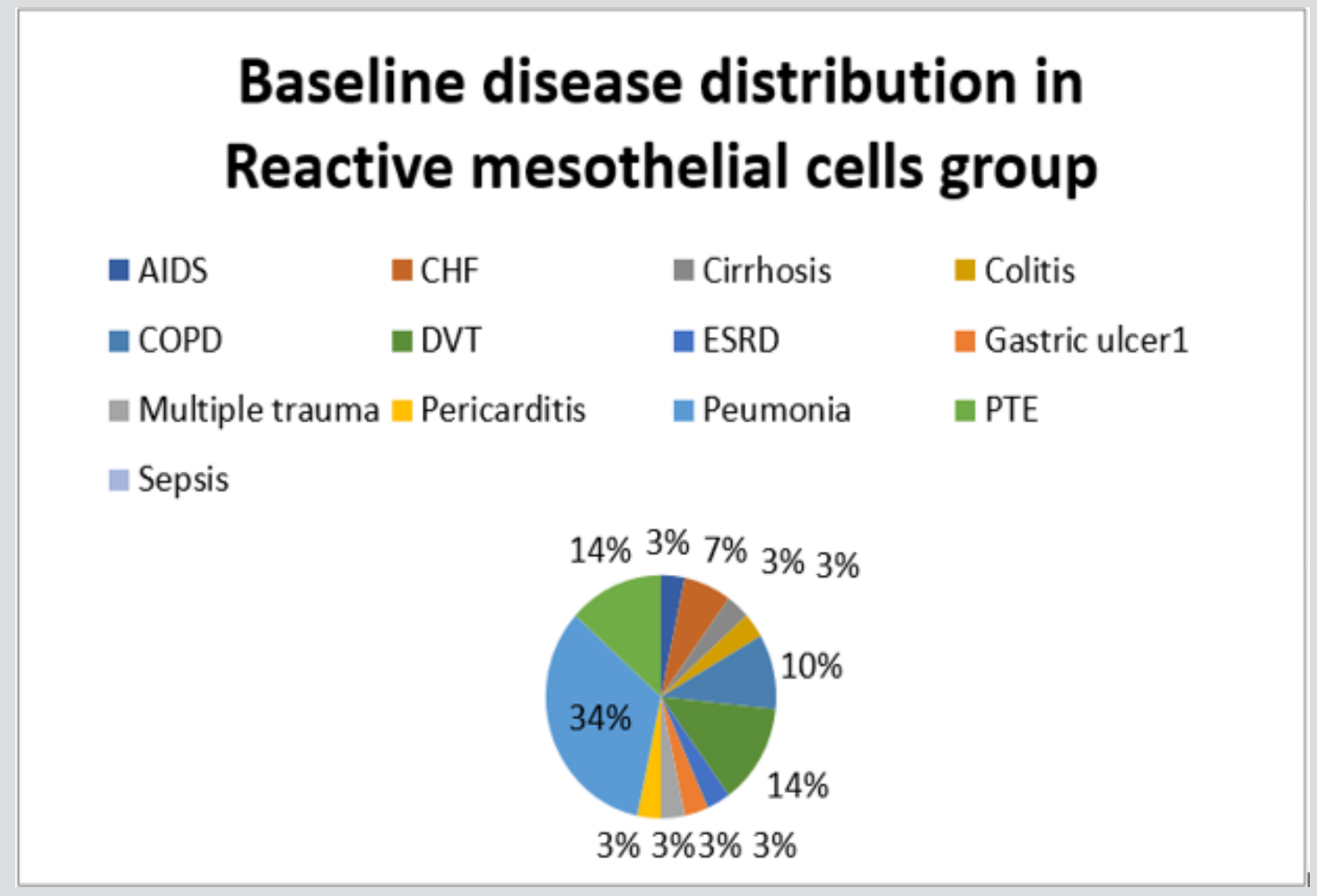

Pie chart 2: Baseline disease distribution in Reactive mesothelial cells group.

Comparison of the means of percentages of different patterns of staining between the two groups revealed that the mean of:

a. Strongly positive staining of p53 in reactive mesothelial cells was zero and in metastatic adenocarcinoma, it was 68.8 (P $=0.001)$;

b. Weakly positive staining of p53 in reactive mesothelial cells was 3.1 and in metastatic adenocarcinoma, it was 31.3 (P $=0.001)$; c. Strongly positive staining of Desmin in reactive mesothelial cells was 71.9 and in metastatic adenocarcinoma, it was $6.3(\mathrm{P}=0.001)$;

d. Weakly positive staining of Desmin in reactive mesothelial cells was 15.6 and in metastatic adenocarcinoma, it was 9.4 (P $=0.001$ ); patterns of p53 and Desmin staining are shown in Figures $1 \& 2$. 


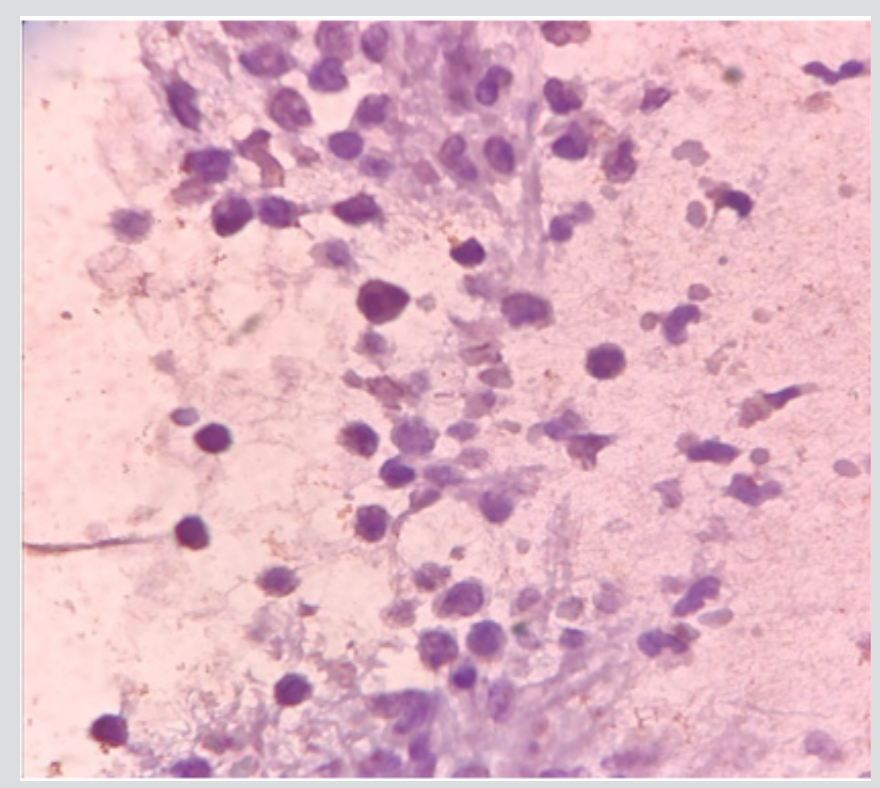

Figure 1: Staining of reactive mesothelial cells with desmin ( $\left.{ }^{*} 1000\right)$.

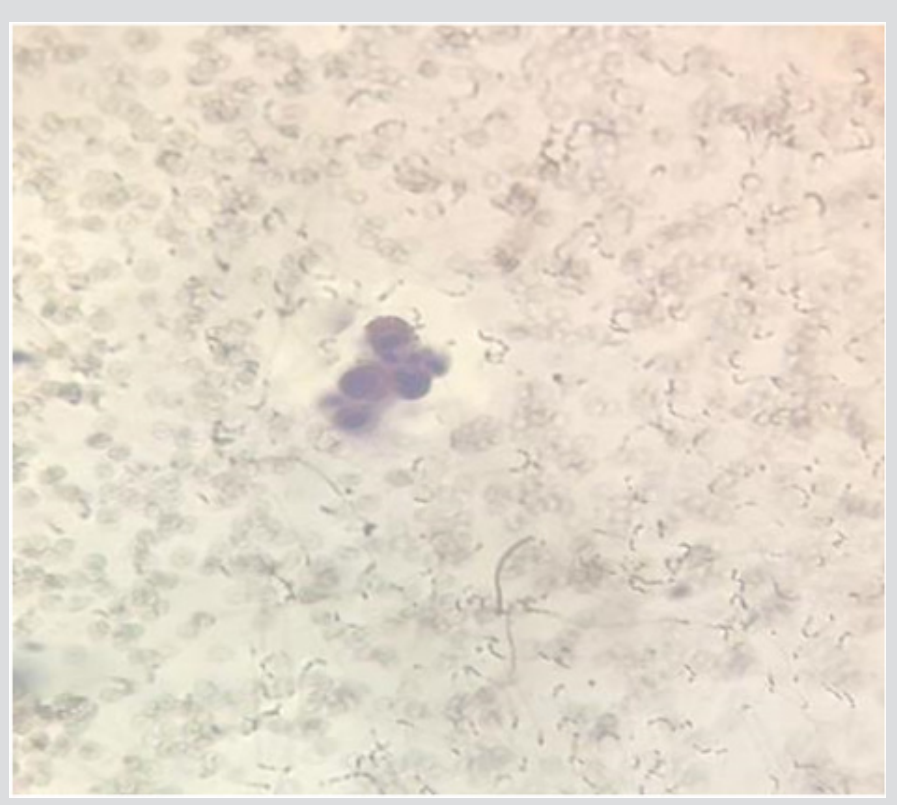

Figure 2: Staining of adenocarcinoma cells with p53(*1000).

\section{Discussion}

In some cases, reactive mesothelial cells may mimic malignant cells feature due to their nuclear changes, including enlargement and irregularity of nuclei with coarse chromatin and conspicuous nucleoli. Clinical data with respect to diseases such as anemia, cirrhosis, systemic lupus erythematosus, pulmonary infarction, renal failure, and AIDS can help in the interpretation of these conditions. Therefore, additional diagnostic procedures are mandatory in these cases [1]. The Tp53 gene is an important suppressor of the tumoral cells and mutant form seen in many cancers. Most mutations occur in a highly protected area, which includes exons 5 to 8 . These exons form the central region of the p53 protein and play a major role in identifying the defective DNA. In the beginning of damage, the TP53 protein level increases in the nucleus of the cell and connects to the DNA. The cell that has the mutated gene of TP53 is no longer able work normally, and perhaps this is the reason why homozygous TP53 loss is a major cause of malignancies [2].

Mutations in this gene is very important, causing a deactivation or defect in the function of this protein, and will make the patient susceptible to creating a wide range. P53 protein is a multivariate transcription factor that contributes to the progression of the cell cycle and the planned cell death process and is associated with several key proteins involved in DNA replication, transcription, and DNA repair [3]. Li-Fraumeni Syndrome are 25 times more 
likely to develop a malignancy and the range of tumors in patients with transient Li-sy syndrome varies greatly. The most common types of tumors are sarcoma, breast cancer, leukemia, brain tumors, and adrenocortical carcinoma. The TP53 gene mutation has been identified in $50-70 \%$ of patients with lung cancer [4]. This study based on molecular methods. In the current study, immunohistochemically detectable p53 was seen in all malignant pleural adenocarcinomas and in few of the reactive hyperplasias. Strongly positive staining of p53 in reactive mesothelial cells was zero and in metastatic adenocarcinoma, it was $68.8 \%$ Weakly positive staining of p53 in reactive mesothelial cells was $3.1 \%$ and in metastatic adenocarcinoma, it was $31.3 \%$.

In a study p53 immunostaining showed nuclear positivity in 31 out of 41 malignant effusions (75.6\%) and for the cases that showed p53 immunostaining only, 9 out of 10 cases (90\%) were malignant [5]. Several studies have suggested that p53 immunostaining does not occur in benign mesothelium but is more common in malignant cells involving serous effusions [6-9]. In a study to differentiate between malignant and benign effusions, p53 immunocytochemical staining had been advocated as a malignant marker in 91 pleural and peritoneal serous effusions. p53 was significantly more expressed in malignant than benign effusions ( $\mathrm{p}<0.0001)$. p53 positivity rate was found in $75.6 \%$ malignant effusions true positive and in $6 \%$ of benign effusions [10]. In another study, mutations in TP53 were reported in patients with head and neck tuberculosis of $60 \%$, which is consistent with the present study due to the positive TP53. In this study, all of the tumor's participants were TP53 positive, which means the TP53 correctly detects all cancer patients [11].

By comparing the protein profiles among adenocarcinoma and reactive mesothelial cells we identified that desmin protein with positive expression in reactive mesothelial cells. In the current study, immunohistochemically detectable desmin was seen in $21.9 \%$ malignant pleural adenocacinomas and in $81.1 \%$ of the reactive hyperplasias. Strongly positive staining of p53 in reactive mesothelial cells was zero and in metastatic adenocarcinoma Strongly positive staining of Desmin in reactive mesothelial cells was $71.9 \%$ and in metastatic adenocarcinoma, it was 6.3\%. Weakly positive staining of Desmin in reactive mesothelial cells was $15.6 \%$ and in metastatic adenocarcinoma, it was $9.4 \%$. In a study the presence of desmin was detected in benign mesothelial cells in 47 of $56(84 \%)$ of reactive cellular specimens and 2 out of $98(2 \%)$ carcinomas specimens [12]. Desmin became the subsequent focus of the study. Desmin is a $52-\mathrm{kDa}$ protein with a wide variety of functions. It is a type III intermediate filament in smooth muscle tissue. It has been confirmed that desmin is one of the earliest protein markers for muscle tissue in embryogenesis as it is detected in the somites of myoblasts.

Desmin is present at low levels during embryogenesis but increases as the cell nears terminal differentiation. Recently studies have shown that desmin may be important in mitochondria function. There is some evidence that desmin may also connect the sarcomere to the extracellular matrix through desmosomes that could be important in signaling between the extracellular matrix and the sarcomere [17]. Now several studies have shown that desmin is a highly sensitive marker for mesothelial cell differentiation and tumor invasiveness in several types of disease including cancer, ESRD, CVD, GI, infection and sepsis disease. Recently proteomics analysis for endometrial carcinoma also discovered the overexpression of desmin in carcinoma tissue. Therefore, desmin was further characterized in this study to confirm the potential variable diagnostic for various disease that analysis revealed that expression of desmin was positive in, ESRD, AIDS, CHF, cirrhosis, colitis, COPD, DVT, gastric ulcer, MT, pericarditis, pneumonia, PTE, and sepsis disease. Therefore, desmin could prove to be a new, sensitive, and specific marker that assists in the detection of various disease. However, more patient is needed to be tested to understand the potential value of this new biomarker.

\section{Conclusion}

It is very important to differentiate between metastatic adenocarcinoma and reactive mesothelial cells in pleural effusions especially overlapping cases which differentiation is critical. The staining with Desmin and p53 are useful in differentiating reactive mesothelial cells from metastatic adenocarcinoma in pleural effusion.

\section{References}

1. Subbarayan D, Bhattacharya J, Rani P, Khuraijam B, Jain S (2019) Use of panel of markers in serious effusion to distinguish reactive mesothelial cells from adenocarcinoma. Journal of cytology 36(1): 28.

2. Toth, Laszlo, et al. (2018) Cell adhesion molecule profiles, proliferation activity and p53 expression in advanced epithelial ovarian cancer induced malignant ascites-Correlation of tissue microarray and cytology microarray. Pathology-Research and Practice 214(7): 978-985.

3. Davidson Ben (2018) Molecular Cytology of Serous Effusions. Molecular Applications in Cytology. Springer Cham pp. 103-115.

4. Li, Qing Kay (2018) Serous Effusion Cytology. Atlas of Non-Gynecologic Cytology. Springer Cham 235-257.

5. Engels M, Michael C, Dobra K, Hjerpe A, Fassina A, et al. (2019) Management of cytological material, pre-analytical procedures and biobanking in effusion cytopathology. Cytopathology 30(1): 31-38.

6. Chen Yao, Nicholas W Mathy, Hongda Lu (2018) The role of VEGF in the diagnosis and treatment of malignant pleural effusion in patients with non-small cell lung cancer. Molecular medicine reports 17(6): 80198030.

7. Leichsenring, Jonas, et al. (2018) Targeted deep sequencing of effusion cytology samples is feasible, informs spatiotemporal tumor evolution, and has clinical and diagnostic utility. Genes, Chromosomes and Cancer 57(2): 70-79.

8. Haase Detlef, Stevenson KE, Neuberg D, Maciejewski JP, Nazha A, et al. (2019) TP53 mutation status divides myelodysplastic syndromes with complex karyotypes into distinct prognostic subgroups. Leukemia!

9. Kelemen Linda E, James D Brenton, David D Bowtell, Brooke L Fridley (2018) Abstract A14: TP53 missense mutations associate with different metabolic pathways A14-A14.

10. Snyder P, Dunbar K, Cipher DJ, Souza RF, Spechler SJ, etal. (2019) Aberrant p53 Immunostaining in Barrett's Esophagus Predicts Neoplastic Progression: Systematic Review and Meta-Analyses. Digestive diseases and sciences 64(5): 1089-1097. 
11. Giacomelli Andrew O, Xiaoping Yang, Robert E. Lintner, James M. Mc Farland, Marc Duby, et al. (2018) Mutational processes shape the landscape of TP53 mutations in human cancer. Nature genetics 50(10): 1381 .

12. Singh Avneesh K, Chan JL, DiChiacchio L, Hardy NL, Corcoran PC, et al. (2018) Cardiac xenografts show reduced survival in the absence of transgenic human thrombomodulin expression in donor pigs. Xenotransplantation 26(2): e12465.

13. Shield PW, Koivurinne K (2018) The value of calretinin and cytokeratin $5 / 6$ as markers for mesothelioma in cell block preparations of serous effusions. Cytopathology 19(4): 218-223.

14. Saleh HA, El Fakharany M, Makki H, Kadhim A, Masood S (2009) Differentiatingreactivemesothelialcells from metastaticadenocarcinoma

\section{ISSN: 2574-1241}

DOI: 10.26717/BJSTR.2019.18.003107

Behnaz Sabaghi. Biomed J Sci \& Tech Res

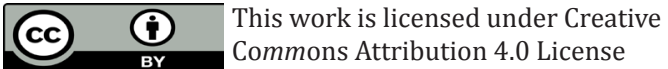

Submission Link: https://biomedres.us/submit-manuscript.php in serous effusions: T0 he utility of immunocytochemical panel in the differential diagnosis. HBME1 Diagn Cytopathol 37(5): 324-332.

15. Szczepulska Wójcik E, Langfort R, Roszkowski Sliz K (2007) A comparative evaluation of immunohistochemical markers for the differential diagnosis between malignant mesothelioma, non-small cell carcinoma involving the pleura, and benign reactive mesothelial cell proliferation. Pneumonol Alergol Pol 75(1): 57-69.

16. Li QK (2018) Serous Effusion Cytology. In Atlas of Non-Gynecologic Cytology. Springer, Cham, pp. 235-257.

17. Pawlak Agnieszka, Rejmak Kozicka E, Gil KE, Ziemba A, Kaczmarek L, et al. (2019) Patterns of desmin expression in idiopathic dilated cardiomyopathy are related to the desmin mRNA and ubiquitin expression. Journal of Investigative Medicine 67(1): 11-19.

$\begin{array}{ll}\text { BIOMEDICAL } & \text { Assets of Publishing with us } \\ \text { RESEARCHES } & \text { - Global archiving of articles } \\ & \text { - Immediate, unrestricted online access } \\ \end{array}$

